\title{
Measuring magnetism in the Milky Way with the Square Kilometre Array
}

\author{
Marijke Haverkorn ${ }^{1,2 *}$, Takuya Akahori ${ }^{3}$, Ettore Carretti ${ }^{4}$, Katia Ferrière ${ }^{5}$, Peter \\ Frick $^{6}$, Bryan Gaensler ${ }^{3,7}$, George Heald ${ }^{8}$, Melanie Johnston-Hollitt ${ }^{9}$, David Jones ${ }^{1}$, \\ Tom Landecker ${ }^{10}$, Sui Ann Mao ${ }^{\dagger 11}$, Aris Noutsos ${ }^{11}$, Niels Oppermann ${ }^{12}$, Wolfgang \\ Reich $^{11}$, Timothy Robishaw ${ }^{10}$, Anna Scaife ${ }^{13}$, Dominic Schnitzeler ${ }^{11}$, Rodion \\ Stepanov $^{6,14}$, Xiaohui Sun ${ }^{3}$, and Russ Taylor ${ }^{15,16}$ \\ ${ }^{1}$ Radboud University; ${ }^{2}$ Leiden University; ${ }^{3}$ The University of Sydney; ${ }^{4}$ CSIRO Astronomy and \\ Space Science; ${ }^{5}$ IRAP, Université de Toulouse, CNRS; ${ }^{6}$ Institute of Continuous Media \\ Mechanics $;{ }^{7}$ ARC Centre of Excellence for All-Sky Astrophysics (CAASTRO); ${ }^{8}$ Netherlands \\ Institute for Radio Astronomy (ASTRON); ${ }^{9}$ Victoria University of Wellington; ${ }^{10}$ Dominion Radio \\ Astrophysical Observatory; ${ }^{11}$ Max-Planck-Institut für Radioastronomie; \\ ${ }^{12}$ CITA; ${ }^{13}$ University of Southampton; ${ }^{14}$ Perm National Research Polytechnic \\ University, ${ }^{15}$ University of Cape Town; ${ }^{16}$ University of the Western Cape \\ E-mail:m.haverkorneastro.ru.nI
}

Magnetic fields in the Milky Way are present on a wide variety of sizes and strengths, influencing many processes in the Galactic ecosystem such as star formation, gas dynamics, jets, and evolution of supernova remnants or pulsar wind nebulae. Observation methods are complex and indirect; the most used of these are a grid of rotation measures of unresolved polarized extragalactic sources, and broadband polarimetry of diffuse emission. Current studies of magnetic fields in the Milky Way reveal a global spiral magnetic field with a significant turbulent component; the limited sample of magnetic field measurements in discrete objects such as supernova remnants and HII regions shows a wide variety is field configurations; a few detections of magnetic fields in Young Stellar Object jets have been published; and the magnetic field structure in the Galactic Center is still under debate.

The SKA will unravel the 3D structure and configurations of magnetic fields in the Milky Way on sub-parsec to galaxy scales, including field structure in the Galactic Center. The global configuration of the Milky Way disk magnetic field, probed through pulsar RMs, will resolve controversy about reversals in the Galactic plane. Characteristics of interstellar turbulence can be determined from the grid of background RMs. We expect to learn to understand magnetic field structures in protostellar jets, supernova remnants, and other discrete sources, due to the vast increase in sample sizes possible with the SKA. This knowledge of magnetic fields in the Milky Way will not only be crucial in understanding of the evolution and interaction of Galactic structures, but will also help to define and remove Galactic foregrounds for a multitude of extragalactic and cosmological studies.

Advancing Astrophysics with the Square Kilometre Array

June 8-13, 2014

Giardini Naxos, Italy

\footnotetext{
${ }^{*}$ On behalf of the SKA Cosmic Magnetism Working Group

† Speaker.
} 


\section{Introduction}

In the past years, cosmic magnetism science has transformed from a niche to a fast growing and exciting field of research. This is largely due to improvements in computational power, and enhanced observational capabilities. The latter led to the presence of extensive new data sets like the Canadian (Landecker et al. 2010) and Southern (Haverkorn et al. 2006a) Galactic Plane Surveys, the Effelsberg 1.4 GHz Medium Latitude Survey (EMLS, Reich et al. 2004), the Urumqi $6 \mathrm{~cm}$ survey (Sun et al. 2007), and the S-band Polarization All-Sky Survey (S-PASS) (Carretti et al. 2015); and ongoing surveys like GALFACTS (Taylor \& Salter 2010) or GMIMS (Wolleben et al. 2009). Unraveling the structure and strength of magnetic fields in the Universe is such a complex and involved system, however, that much is still under debate or plainly unknown.

Studies of magnetic fields in galaxies are at the center of many open questions regarding cosmic magnetism. Current galactic magnetic fields may originate in the primordial universe, possibly from magnetizing topological defects, from early stellar magnetic fields (Widrow 2002) or from magnetic fields in the cosmic web (Sofue et al 2010). Seed fields may also be generated in protogalaxies by e.g. the Biermann mechanism in the first supernova remnants (Hanayama et al. 2005), the Weibel instability (Lazar et al. 2009), or from plasma fluctuations (Schlickeiser \& Felten 2013). Magnetic fields in the intracluster and intergalactic medium may have originated from outflows of galaxies (Donnert et al. 2009). On smaller scales, galactic magnetic fields interact with supernova remnants, and help shape fields in star formation regions (Fish et al. 2003; Green et al. 2012).

The Milky Way is an ideal testbed for many galactic magnetic field studies due to its proximity. We have the most detailed observations and understanding of the multi-phase interstellar environment in the Milky Way to help interpret magnetic field studies. In particular, for magnetic fields in discrete objects and on smaller scales $(\lesssim 1 \mathrm{pc})$, the Milky Way is the best location where these can be studied in detail (although parsec-scales become accessible in the Magellanic Clouds as well, see Beck et al (2015)). The global configuration of the magnetic field and characterization of magnetic fields in the gaseous halo is difficult to determine from our location within the Milky Way, and is more easily studied in other (face-on) spiral galaxies. However, the Milky Way provides the opportunity of studying large-scale reversals in this global configuration of the field in unique detail.

In addition, the Milky Way is a significant foreground for a wide variety of extragalactic studies. The magnetic fields in the Milky Way disk and halo provide a characteristic radio-polarimetric pattern, which needs to be understood to enable study of e.g. the Epoch of Reionization, inflation theory through the Cosmic Microwave Background B-mode polarization, or magnetization of the cosmic web. Also, knowledge of the structure of the Galactic magnetic field is imperative to trace back Ultra-High Energy Cosmic Rays to their sources, and to understand the multi-phase, multi-component interstellar medium (ISM).

In the Square Kilometre Array (SKA) Science Case written in 2004, Beck \& Gaensler (2004) described SKA observations with magnetic fields in the Milky Way and nearby galaxies, and stated the importance of an all-sky Rotation Measure (RM) Grid of compact extragalactic radio sources. In the updated SKA Science Case for magnetic fields in the Milky Way presented in this chapter, we re-emphasize the importance of the RM Grid, but in addition explain the necessity of broadband 
polarimetry of diffuse synchrotron emission, enabling Faraday Tomography through RM synthesis (Brentjens \& de Bruyn 2005).

Section 2 details the current status of research in magnetic fields in the Milky Way, from the global configuration and turbulent fields, to fields in discrete objects and in the Galactic Center. In Section 3, we describe the Galactic magnetism studies that will become possible with the SKA. Subsection 3.1 details the two methods used, viz. the RM Grid and Faraday Tomography, whereas in the next three subsections we present the possibilities with SKA in phase 1 Early Science, SKA in phase 1 and SKA in phase 2. .

\section{Magnetic Fields in the Milky Way}

Galactic magnetic fields are amplified and maintained by magnetic dynamo action, through conversion of energy from e.g. differential rotation, turbulence and/or cosmic rays into magnetic energy. Regular fields may be amplified by mean-field, alpha-omega dynamos, which are extensively studied both analytically and numerically. Various dynamo modes may exist, resulting in characteristic configurations of the global galactic field. Specific dynamo modes may indicate the influence of an external disturber or of a bar (Ferrière 2007). In galactic disks, the dynamo mode most easily excited is axisymmetric and has even parity with respect to the midplane (i.e., the horizontal field component has the same direction on either side of the midplane, while the vertical component changes sign across the midplane (e.g., Ruzmaikin et al. 1988)). The field is dominated by its toroidal component, which together with the radial component, forms an axisymmetric spiral pattern. Rarely observed bisymmetric fields in galactic halos could be relics of the initial intergalactic magnetic field (as suggested by Fletcher et al. (2011) for the halo of M51). As opposed to flat disks, dynamos in spherical objects - such as galactic halos - are expected to favor the axisymmetric mode with odd vertical parity, in which the magnetic field crosses the midplane continuously at right angles and the horizontal component reverses direction across the midplane ${ }^{1}$.

In the disk and halo system of the Milky Way, this would result in mixed-parity modes (Sokoloff \& Shukurov 1990). Although Moss \& Sokoloff (2008) find that in a disk-halo system, usually the stronger dynamo enslaves the other, resulting in either an even-parity or an odd-parity magnetic configuration, mixed-parity modes can be obtained if a moderate Galactic wind is included (Moss et al. 2010). The geometry and parity of the halo magnetic field hold the key to its origin. For instance, an X-shaped field would probably indicate the existence of a large-scale Galactic wind. Moreover, even parity of this X-shaped field would suggest a wind originating near the Galactic plane and advecting the dynamo field of the disk into the halo, while odd parity would suggest a wind blowing from the base of the halo and stretching out the (normally dipole-like) dynamo field of the halo into an X shape (Ferrière \& Terral 2014).

Current observations show a picture generally consistent with dynamo models, albeit so rough that it is impossible yet to determine how exactly the Galactic magnetic field is maintained. It is now well-established that the large-scale magnetic field in the Milky Way disk is generally aligned with the spiral arms and has a strength of about $3 \mu \mathrm{G}$ (see the review by Haverkorn (2014)). However, there is still considerable debate over a few basic properties of the large-scale field, including

\footnotetext{
${ }^{1}$ Note that the even-parity poloidal field is also said to be quadrupolar, and the odd-parity poloidal field is also named dipolar, although it generally differs from a pure quadrupole and dipole, respectively.
} 
its azimuthal structure (i.e., whether it is axisymmetric, bisymmetric, or neither as concluded by Men et al. (2008)), and the number and location of large-scale reversals in the field direction. Most recent models based on multiple observational tracers favor an axisymmetric spiral structure with a single reversal just inside the Solar circle (Brown et al. 2007; Sun et al. 2008; Van Eck et al. 2011; Jansson \& Farrar 2012). However, some of the models based on only pulsar data conclude that multiple reversals must exist (Han et al. 2006; Nota \& Katgert 2010). In principle, large-scale field reversals can be produced by dynamos when the rotational velocity has a large vertical gradient (Ferrière \& Schmitt 2000), or be a result of early injection of small-scale fields (Hanasz et al. 2009; Moss et al. 2012) or of magneto-rotational instability (Machida et al. 2013). Much of the controversy about the presence of large-scale field reversals in the Milky Way is fueled by the lack of detection of field reversals in external galaxies, with the exception of M 81 which appears to host a bisymmetric field (see Beck et al (2015) for a detailed discussion).

Recent Galactic magnetic field models seem to confirm the mixed parity dynamo modes in disk and halo (Frick et al. 2001; Sun et al. 2008; Jansson \& Farrar 2012). This also would explain the apparent contradiction between the observed "butterfly pattern" of rotation measures in the inner Galaxy, indicating a reversal of the azimuthal field component across the Galactic plane (SimardNormandin \& Kronberg 1980) and local measurements of field direction indicating no reversal of this component across the galactic plane (Frick et al. 2001). The first would be associated with the halo field, whereas the second reflects the disk field. However, characterizing the global halo field structure is made difficult by the contamination of local structures. As an example, the Faraday rotation pattern of extragalactic sources recognized as a large-scale dipolar field in previous studies is strongly influenced by a local magnetized bubble (Wolleben et al. 2010; Sun \& Reich 2010). Several forms of the halo magnetic field geometry have been proposed in the literature, including a purely azimuthal double-toroidal component (e.g., Sun \& Reich (2010)), a spiral component (e.g., Mao et al. (2012)) and an out-of-plane X-shaped component (e.g., Jansson \& Farrar (2012)) as observed in external edge-on spiral galaxies and as modeled by Ferrière \& Terral (2014). Vertical field components are expected in the Milky Way, by analogy to nearby spirals (Beck et al 2015). However, the vertical component of the field at the Galactocentric radius of the Sun is observed to be small (Mao et al. 2010). This suggests that the Milky Way's vertical field components are concentrated towards the inner Galaxy, as modeled in e.g. Jansson \& Farrar (2012).

The observations on which these magnetic field models are based consist of RM Grids of extragalactic sources and pulsars, and/or diffuse synchrotron emission. The models are hampered by low density of polarized sources, uncertain distance estimates of pulsars, and contamination by foreground structures such as supernova remnants (e.g. the radio loops). A good example of this is the wavelet analysis of pulsar data to determine the large-scale structure of the Galactic magnetic field (Frick et al. 2001), which shows good results in the Solar neighborhood, but cannot determine the global field structure due to insufficient data points (Stepanov et al. 2002). This is illustrated in the left plot of Figure 1, which shows a wavelet transform of a test function representing Galactic electron density obtained from an irregular grid of pulsar data (Stepanov et al. 2002). The plot demonstrates the method's unique capability to trace out magnetic spiral arms, but also that this is currently only possible in the Solar vicinity. 

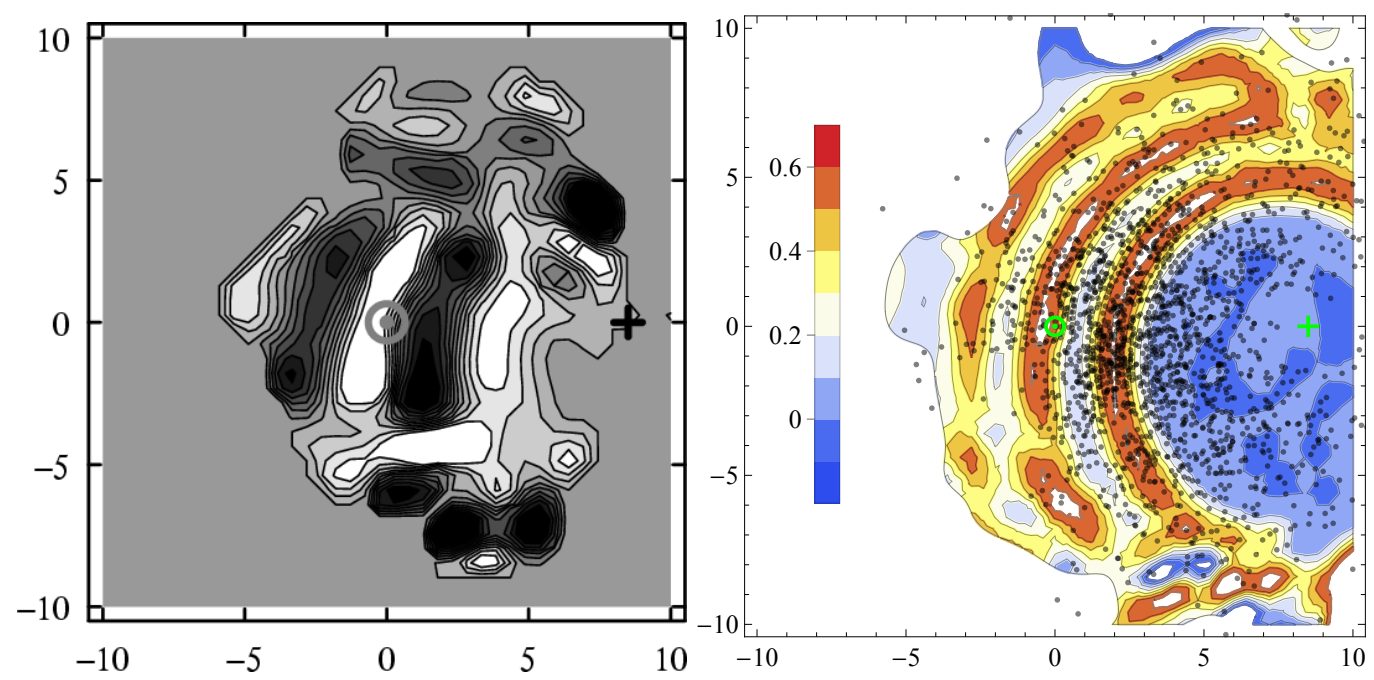

Figure 1: Wavelet transforms of a test function containing a Galactic electron density model, retrieved from pulsar RMs. The Galactic Center is marked with a cross and the Sun is at the center of the frames. The axes are in $\mathrm{kpc}$, and in uniformly grey/white regions, data is too sparse. Left: current situation, using 323 pulsars with known RMs (Stepanov et al. 2002) and a two-arm model. Right: the same with a simulated pulsar data set of 2000 pulsars to be expected with SKA1, fitting well to a three-arm model.

\subsection{Galactic turbulence}

Both ionized and neutral phases of the Galactic ISM are turbulent, usually displaying a Kolmogorovlike power spectrum in density across a wide range of length scales (Elmegreen \& Scalo 2004; Armstrong et al. 1995). Determining the power spectrum of the magnetic field is much more difficult: reliable estimates only exist for related quantities like RM. Observed spectral indices ${ }^{2}$ of RM are generally much flatter than Kolmogorov (Haverkorn et al. 2008).

Besides the spectral index, magnetohydrodynamic (MHD) turbulence can be characterized by several fundamental parameters including scales of energy injection and dissipation, the rms Mach number of flow speed, intermittency, and the plasma beta (the pressure ratio of the gas to the magnetic field). The search for such characteristics is important to understand the dynamical and thermal states of the ISM. In addition, numerical modeling of turbulence is essential to understand the physics behind the observations of turbulence parameters. E.g., modeling revealed that known turbulent structures are not enough to explain observed structure functions ${ }^{3}$ (SFs) of RM toward high Galactic latitudes (Akahori et al. 2013). A possible cause of the discrepancy would be magnetic fields emerging from the disk to the halo due to the magneto-rotational and Parker instabilities (Machida et al. 2013).

The Mach number of interstellar turbulence can be determined through polarization data, by way of the gradient of the polarization vector (Gaensler et al 2011). Application of this method

\footnotetext{
${ }^{2}$ Usually, power in RM fluctuations is measured by structure functions (as explained below rather than power spectra, the slope of which is directly related to power spectral index.

${ }^{3}$ The structure function of order $n$, of a quantity $f$ as a function of scale $\delta \mathbf{x}$ is defined as $\operatorname{SF}_{f}(\delta \mathbf{x})=\langle| f(\mathbf{x})-f(\mathbf{x}+$ $\left.\delta \mathbf{x})\left.\right|^{n}\right\rangle_{\mathbf{x}}$, where \langle\rangle$_{\mathbf{x}}$ denotes averaging over all positions $x$. The second-order structure function $(n=2)$ is most used and usually meant when just "structure function" is used.
} 


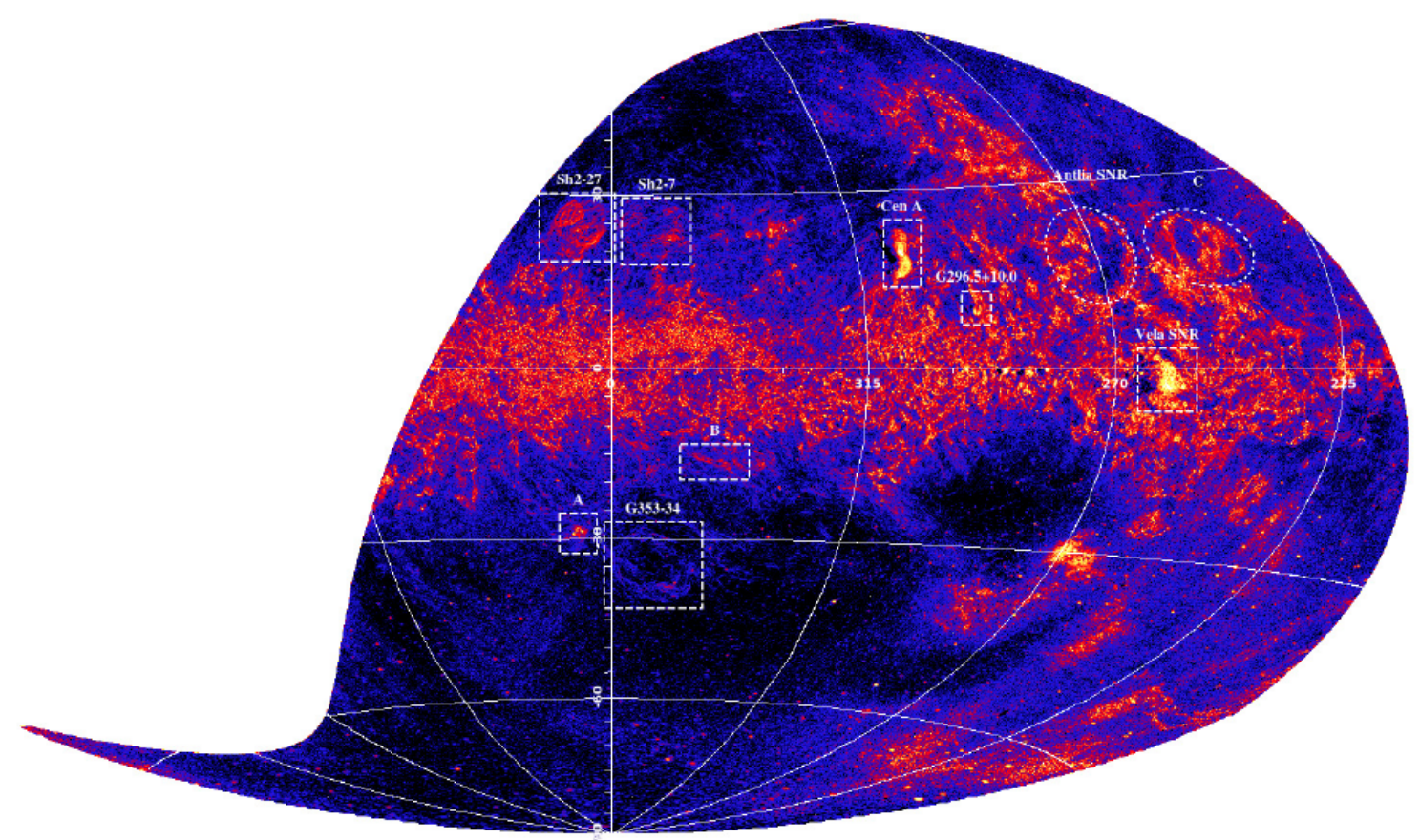

Figure 2: Gradient of the polarization vector over the entire southern sky (Iacobelli et al. 2014). Analysis of the probability distribution function of the polarization gradient shows that interstellar turbulence is transonic in the regions studied.

on a small test region, combined with simulations (Burkhart et al. 2012), reveals that interstellar turbulence is transonic with $M \lesssim 2$. Iacobelli et al. (2014) showed that this Mach number value is roughly consistent over the entire (Southern) sky, as can be derived from the polarization gradient shown in Figure 2.

Measured maximum scales of fluctuations in the magneto-ionized medium are likely to be connected to the scale of energy injection in the interstellar gas. Energetics imply that the main energy sources likely to drive interstellar turbulence are supernova remnants. Early observational studies using correlation functions corroborated this, concluding that the outer scale of turbulence must be $O(100)$ pc (Lazaryan \& Shutenkov 1990). Usually, these maximum scales are probed by second-order SFs of RM, to be obtained from measurements of RM Grids. However, recent observations indicate maximum scales of fluctuations smaller than $\sim 10 \mathrm{pc}$ (in spiral arms only, Haverkorn et al. 2008), of a few parsecs from anisotropies in $\mathrm{TeV}$ cosmic ray nuclei (Malkov et al. 2010), and similar from analysis of synchrotron fluctuations (Iacobelli et al. 2013). These small scales do not necessarily indicate the maximum scale of turbulence, but only show that larger-scale fluctuations in the magneto-ionic medium are not observed. Turbulence may still be present on scales up to $\sim 100 \mathrm{pc}$, but this structure may be masked by small discrete sources (Haverkorn et al. 2006b), strong small-scale fluctuations in electron density, dominating shock waves in spiral arms, etc. The SF at higher Galactic latitudes has a flatter slope in $>1^{\circ}$ scales (Stil et al. 2011). These authors claim that the steep slope of SFs at low latitude could be affected by the local turbulent ISM. 
Some evidence exists that interstellar turbulence in dense gas is intermittent, i.e. contains regions with intense turbulence and regions with no turbulence, giving long tails in the distribution of the observable such as density or velocity (Elmegreen \& Scalo 2004). This can be detected using higher order SFs. However, in the diffuse ionized gas, current SFs contain far too few sources to be able to construct meaningful higher order SFs.

\subsection{Magnetic fields in discrete objects}

Star forming regions The launching mechanism for jets in general has been proposed to be universal (e.g. Livio (2011)), crossing orders of magnitude in terms of source energetics and environmental conditions. However, our observational understanding of jets in Young Stellar Objects (YSOs) is almost diametrically opposed to that of their higher energy analogues such as AGN, GRBs and X-ray binaries. While characteristic quantities such as density, temperature and ionization fraction can be well constrained for YSOs from (primarily) line emission data, the strength and orientation of the magnetic field in these systems is still very poorly known (Ray 2009). Observational evidence for these magnetic fields is generally indirect and model dependent: jet rotation, cushioning effects on jet shocks, etc. Although non-thermal radio emission can provide a direct measure of magnetic field strength and configuration, measurements of these properties are very rare (Ray et al. 1997; Carrasco-González et al. 2010; Ainsworth et al. 2014). This is because the associated non-thermal emission is faint $(\sim \mu \mathrm{Jy})$, and because radio studies of star forming regions are predominantly total intensity, $\mathrm{cm}$-wave experiments focusing on thermal radio emission from ionized gas.

In spite of this, the role of the magnetic field in jet launching is expected to be inextricably linked to the active accretion of the central object from its gaseous disk and surrounding molecular cloud, through which Galactic magnetic field lines are known to be threaded and where the influence of the magnetized jet may provide turbulent support against collapse. The centrifugal acceleration of material along these field lines is widely accepted to be closely connected to the launching of YSO jets through MHD processes, as well as the collimation of those jets through a "winding-up" of the magnetic field lines anchored in the rotating disk leading to the creation of hoop stresses in the resulting helical field configuration (Blandford \& Payne 1982; Ferreira et al. 2006). Furthermore, the magnetic fields in these systems are crucial not only for understanding the star formation process but, through the acceleration of relativistic particles in jet shocks, also act as cosmic-ray factories, contributing to the GeV excess in the Galaxy (Orlando \& Strong 2013). ${ }^{4}$

Supernova remnants Magnetic fields in supernova remnants (SNRs) can be enhanced to $\mathrm{mG}$ strengths. Magnetic field strengths in young SNRs are generally radially directed, whereas old SNRs have mostly tangential fields, although turbulent fields play a significant role (for a review see Reynolds et al. (2012)). Apart from the importance of magnetic fields for the evolution of SNRs themselves, these fields are crucial for acceleration of Galactic cosmic rays (Blasi 2013). SNRs are also believed to be the main drivers of interstellar turbulence.

There is some evidence from both observations (Gaensler 1998; Uyaniker et al. 2002; Kothes \& Brown 2009) and numerical simulations (Stil et al. 2009) that magnetic fields in SNRs carry

\footnotetext{
${ }^{4}$ For SKA measurements of magnetic fields in molecular and cold gas, linked to the early phases of star formation, we refer the reader to Robishaw et al. (2015).
} 
the signature of larger-scale Galactic magnetic fields which they are expanding in. Similarly, in some SNRs, there is evidence for toroidal fields, interpreted as the product of the stellar wind of the progenitor, swept up by the blast wave (Uyaniker et al. 2002; Harvey-Smith et al. 2010). Some massive stars exhibit non-thermal radio emission, so magnetic fields must occasionally be present in the winds. Nevertheless, de la Chevrotière et al. (2014) failed to find optical evidence of magnetic fields in $11 \mathrm{WR}$ stars (by searching for circular polarization). Only a large sample of SNR magnetic fields will shed light on the relationship between magnetic fields in SNRs and stellar and/or Galactic magnetic fields.

Measurements of magnetic fields in Pulsar Wind Nebulae (PWNe) are scarce and biased towards young, bright PWNe. However, an intriguing example of the importance of observing PWN magnetic fields is given by Kothes et al. (2008), who showed that the older PWN DA 495 has a dipole-like field, giving an estimate of the spin axis of the putative pulsar. The PWN magnetic field configuration is best studied through depolarization and Faraday tomography of the PWN emission itself, but probing through RMs of background sources can contribute. For more discussion on PWN science with the SKA, see Gelfand et al. (2015).

For a discussion on other SNR science with the SKA, see Wang (2015).

HII Regions Typical magnetic field strengths in HII regions are a few to about $12 \mu \mathrm{G}$ (Sun et al. 2007; Gao et al. 2010; Harvey-Smith et al. 2011), obtained from observations of the Faraday rotation of background Galactic synchrotron emission. Magnetic field measurements in HII regions over a large range of densities should be obtained to understand the dynamic role that magnetic fields play. This can be done using a dense RM Grid. Strömgren spheres tend to be so dense and so turbulent that they are Faraday thick at $1 \mathrm{GHz}$, so that emission from the objects themselves can only be probed at higher frequencies, where the regions become Faraday thin.

Magnetic fields in HII regions also seem to retain knowledge about larger-scale Galactic magnetic fields (Han \& Zhang 2007; Harvey-Smith et al. 2011). In particular, this should be true of Planetary nebulae (PNe), which do not have an intrinsic magnetic field. However, some have recently been shown to have a polarization signature (Ransom et al. 2008, 2010). Therefore, mapping out magnetic field directions in PNe gives an independent probe of the large-scale field. Extensive catalogues exist of PNe, down to arcsecond size objects. Measurements of the Faraday rotation in the ionized shell can be coupled with optical observations which can supply data on the distance, the size of the ionized region, and the electron density. This can lead to the determination of unambiguous magnetic field values all over the Galaxy.

Faraday screens Faraday screens are structures observed through their polarization properties (Gray et al. 1998; Haverkorn et al. 2003; Wolleben \& Reich 2004), which are difficult to identify as physical Galactic objects from turbulent structures. These objects are invisible or at best very faint in the continuum, but visible in polarized emission and RM. Some of them can be described as magnetic bubbles with strong regular fields and low thermal electron density, when their magnetic pressure exceeds thermal pressure. At $4.8 \mathrm{GHz}$, Faraday screens with high RMs of the order of $200 \mathrm{rad} \mathrm{m}^{-2}$ were identified in the Galactic plane in polarized emission (Sun et al. 2007; Gao et al. 2010), whereas lower-frequency data reveal screens of a few to a few tens of $\mathrm{rad} \mathrm{m}^{-2}$. Many more Faraday screens are expected to have lower RMs, but these are easily be masked by small-scale 
polarization from the magnetized turbulent ISM. Some of them may be linked to faded PWNe or old HII regions leaving expanding magnetic bubbles (Iacobelli et al. 2013), but in general their nature and origin are still enigmatic.

\subsection{The Galactic Center}

The Galactic Center (GC) is important - aside from its intrinsic interest - because it is, by definition, the closest example of a galactic nucleus that we possess. It has a complex magnetic field structure in interaction with the gas and relativistic particles close to the GC (see Ferrière (2009) for a review). The GC magnetic field is most likely a result of dynamo action, either a local dynamo or the dynamo maintaining the Galactic magnetic field, which may require a central galactic wind to stabilize (Moss et al. 2010). Crocker et al. (2011) showed evidence that a GC wind is indeed present. Therefore, measuring and understanding magnetic fields in the GC is also a key component in understanding Galactic magnetic fields.

Large uncertainties still exist about the field strength. Estimates are $\sim(6-22) \mu \mathrm{G}$ (minimum energy), $\gtrsim(50-120) \mu \mathrm{G}$ (synchrotron spectral break, Crocker et al. $(2010,2013))$ or $\sim 1 \mathrm{mG}$ (pressure balance) in the general intercloud medium. Measurements of the RM and dispersion measure $(\mathrm{DM})$ of the magnetar J1745-2900 indicate that the magnetic field at $\sim 0.1 \mathrm{pc}$ from Sgr A* could be as high several $\mathrm{mG}$ (Eatough et al. 2013). Zeeman measurements and submm polarimetry in dense clouds show field strengths of $0.1 \mathrm{mG}$ to a few $\mathrm{mG}$ (e.g., Killeen et al. 1992; Crutcher et al. 1996; Chuss et al. 2003).

The configuration and structure of the GC magnetic field are also significantly unclear. Novak et al. (2003) and Law et al. (2011) used a RM Grid to obtain a magnetic field geometry organized on $\sim 300 \mathrm{pc}$ scales, centered some $50 \mathrm{pc}$ west of the dynamical center of the Galaxy. They interpret polarization in radio synchrotron filaments as a poloidal (vertical) field in the diffuse ISM. This is in conflict with the findings of Roy et al. $(2005,2008)$, who obtained results - based on an RM Grid of 64 background sources - consistent with a bi-symmetric spiral magnetic field in the Galaxy or with a field oriented along the central bar of the Galaxy, with a field strength of $\sim 20 \mu \mathrm{G}$.

Unanswered questions also remain about how the recently-discovered Fermi Bubbles are related to the central regions of our Galaxy: are they the result of electron-dominated processes ( $\mathrm{Su}$ et al. 2010) from close to the $\sim 4.1 \times 10^{6} M_{\odot}$ black hole at the center, or are they formed by proton dominated objects (Crocker \& Aharonian 2011) arising from the stellar activity in the central $\sim 200$ pc? Evidence for the latter seems to have emerged from polarized radio counterparts to the Fermi bubbles detected in the S-PASS survey (Carretti et al. 2013). The magnetic field structure is a critical part to the resolution of this question, since the field structure is likely intimately tied to both the maintenance of the dynamo and the formation of Bubble-like structures in galaxies through the establishment of winds.

\section{Galactic Magnetism Science with the Square Kilometre Array}

\subsection{Methods}

Although the Galactic Science described in this chapter is very diverse, the science goals can be reached using two main observational strategies, viz. the RM Grid and Faraday tomography with 
broadband polarimetric observations of diffuse Galactic radio emission. We describe these methods in this Section, and then explain how we can reach our science goals with these observations in the SKA1 phase, SKA1 Early Science and in SKA2.

\subsubsection{The RM Grid}

The RM Grid as described in the 2004 SKA Science Case (Beck \& Gaensler 2004) continues to be the highest priority for the Cosmic Magnetism Working Group (see Johnston-Hollitt et al. (2015)). The RM Grid possible with SKA1 is based on the summary of the Cosmic Magnetism Science Working Group Assessment Workshop (Govoni et al. 2014). These observations focus on a relatively shallow spectro-polarimetric survey at $\sim 1 \mathrm{GHz}$ over a broad frequency coverage, enabling a vastly improved RM Grid. Currently, about 40,000 extragalactic background sources with measured RMs are available in the RM Grid, mostly based on Taylor et al. (2009). A SKA1SUR Band 2 survey with $2 \mu \mathrm{Jy}$ sensitivity (4 hours per pointing with $500 \mathrm{MHz}$ bandwidth) may provide RM Grids of 300-1000 sources/ $\mathrm{deg}^{2}$, i.e. an improvement of $2-3$ orders of magnitude to the current status.

Pulsar RM surveys also offer a vast improvement in data quality. Smits et al. (2011a) estimate that SKA1-LOW will provide 9,000 - 10,000 new pulsar detections, including their RMs, whereas SKA1-MID will discover about 12,000 - 13,000. Recent estimates predict that 30,000 new pulsars will be discovered with the SKA, 50\% of which will already be found with SKA1 (Kramer \& Stappers 2015). These pulsar measurements become exceptionally valuable for modeling the Galactic magnetic field when reliable distance estimates are available. For this goal, the step to SKA2 will be crucial: in SKA2, timing parallaxes should allow distance measurements with an accuracy of about $5 \%$ up to a maximum distance of $30 \mathrm{kpc}$, which means throughout the Milky Way (Smits et al. 2011b). See Han et al. (2015) for more detailed discussion of the use of pulsars to investigate the magneto-ionic medium of the Milky Way.

\subsubsection{Faraday tomography}

In the past few years, Faraday tomography ${ }^{5}$ has started to provide new views into the structure of the Galactic magnetic field, albeit still incomplete and puzzling. The few studies available for various sightlines all find that the path length is filled with a small number of discrete synchrotron emission regions, interspersed with Faraday-rotating screens (Schnitzeler et al. 2007; Brentjens 2011; de Bruyn \& Pizzo 2014; Jelić et al. 2014). This hints at the complexity of the magnetic field structure, but is at least partially due to the insensitivity of current observations to Faraday-thick structures.

The greatest leap in knowledge about Galactic magnetism may well lie in the opportunities that Faraday tomography will give. Long wavelengths are required for a high maximum detectable Faraday thickness of $\Delta \phi_{\max }=\pi / \lambda_{\min }^{2}$, while broad frequency coverage provides a high Faraday depth resolution $\delta \phi=2 \sqrt{3} / \Delta \lambda^{2}$ (Brentjens \& de Bruyn 2005). Typical Faraday depths in the tomography studies above are a few to a few tens of $\mathrm{rad} \mathrm{m}^{-2}$, while objects like SNRs or PWNe show Faraday depths up to a few hundred $\mathrm{rad} \mathrm{m}^{-2}$. Therefore, a Faraday tomography survey

\footnotetext{
${ }^{5}$ For an extended explanation, see Heald et al. (2015).
} 
needs high Faraday depth resolution and capability to observe Faraday thick structures (up to $\sim$ $\left.100 \mathrm{rad} \mathrm{m}^{2}\right)$, necessitating broad frequency coverage at relatively low frequencies $(<1 \mathrm{GHz})$.

The broad frequency coverage needed for Faraday tomography is a key aspect for the broadband polarimetry project described by Gaensler et al. (2015). Even though their science goals focus on Faraday tomography of extragalactic radio sources themselves, the data can be taken commensally for the two projects. The parameters needed for their survey match well with the Galactic science goals described here.

\subsection{Prospects for SKA1-Early Science}

For the SKA1 Early Science phase, we intend to use the results from a combined all-sky polarimetric survey with SKA1-SUR Band 2 with the ASKAP-POSSUM survey (Gaensler et al. 2010) as proposed by Gaensler et al. (2015). These combined data would have continuous frequency coverage from $700 \mathrm{MHz}$ to $1500 \mathrm{MHz}$, which translates into a maximum detectable Faraday thickness $\Delta \phi_{\max } \approx 80 \mathrm{rad} \mathrm{m}^{-2}$ and a Faraday depth resolution $\delta \phi=24 \mathrm{rad} \mathrm{m}^{-2}$.

For studies of the ordered and random components of the Galactic magnetic field, the Faraday depth resolution of this Early Science survey is too low, although analysis techniques like QUfitting (O'Sullivan et al. 2012) can increase the Faraday depth resolution significantly.

However, this survey is well suited to study discrete objects like SNRs, which have higher observed RMs. In research on SNRs and other discrete objects, the SKA Pathfinders will already make big advances, given their ability to image SNRs at the other side of the Galaxy (10" resolution at a distance of $15 \mathrm{kpc}$ gives a physical resolution of $1 \mathrm{pc}$ ). This will allow some sense of magnetic field strength in these objects.

However, the proposed combined SKA1 Early Science polarimetric survey will be able to map magnetic field strengths in SNRs. This will increase the number of SNRs with known magnetic field structure by orders of magnitude, finally making statistical studies of SNR magnetic fields possible. Faraday depths within a SNR have been found to vary by $100-200 \mathrm{rad} \mathrm{m}^{-2}$ (Uyaniker et al. 2002; Harvey-Smith et al. 2010). If these Faraday thick components are uniform, this survey will not be able to detect them. However, the Faraday depth maps show ample small-scale structure, making it plausible that a large part of the Faraday depth can be detected. Any missing large-scale Faraday depth may be estimated from the RM Grid. Assuming that we want to probe such a SNR in 10 Faraday depth slices, the proposed survey's sensitivity of $\sim 7 \mu \mathrm{Jy} \mathrm{bm}^{-1}$ at a resolution of $10^{\prime \prime}$ will increase the number of SNRs for which we have magnetic field measurements from a few to a few hundred ${ }^{6}$.

\subsection{Prospects for SKA1}

With the increased sensitivity of SKA1 with respect to the Early Science phase, weaker magnetic fields will become visible, such as the interstellar magnetic fields in the Galactic plane and halo, using both broadband polarimetry of diffuse emission and Faraday tomography. Again we suggest to commensally use the proposed Broadband Polarimetry Survey for SKA1 (Gaensler et

\footnotetext{
${ }^{6}$ Compare to current capabilities of e.g. the Canadian Galactic Plane Survey SNR catalog (Kothes et al. 2006), where 24 SNRs were detected in polarization (single channel) at $1420 \mathrm{MHz}$, over $110^{\circ}$ of the outer Galactic plane, with a sensitivity of $\sim 5 \mathrm{mJy} \mathrm{bm}^{-1}$.
} 
al. 2015), which will have full frequency coverage over a range of $650-1750 \mathrm{MHz}$ at $1^{\prime \prime}$ resolution (ten times the resolution at Early Science) over the whole sky down to a sensitivity of $\sim 5 \mu \mathrm{Jy} \mathrm{bm}^{-1}$. The high resolution and sensitivity can be used to unveil the non-thermal emission in star forming regions, and probe SNRs and HII regions to larger distances.

However, detailed mapping of the interstellar magnetic field will need coverage at lower frequencies to obtain sufficient Faraday depth resolution. Assuming that Band 1 will be available for SKA1-SUR, an all-sky survey in the $350-650 \mathrm{MHz}$ range to complement the SKA1-Early Science survey is needed to provide a Faraday depth resolution of $\delta \phi=5 \mathrm{rad} \mathrm{m}^{-2}$. As detailed in Figure 7 in Braun (2013), a 10"'-resolution all-sky survey down to $\sim 7.6 \mu \mathrm{Jy} \mathrm{bm}^{-1}$ across $350-650 \mathrm{MHz}$ would imply 2 full years of observing time. Therefore, obtaining these low frequencies down to the sensitivity of the proposed SKA1-SUR Band 2 survey would be better as a SKA2 project (see below).

Galactic magnetic fields In order to assess the possibilities of observing Galactic turbulent magnetic fields with SKA, simulations of high-resolution SKA observations were made of total intensity and polarized Galactic emission in various directions of the sky within the SKADS program (Sun \& Reich 2009). Based on realistic Galactic 3D-models of the distribution of thermal electrons, cosmic-ray electrons, and magnetic fields (Sun et al. 2008), 1.6" resolution maps at $1.4 \mathrm{GHz}$ for total and polarized intensities and RMs were calculated, similar to the proposed survey above. The extent of diffuse structures depends on the outer scale of the assumed Kolmogorov spectrum for the turbulent magnetic field, the length of the line-of-sight through the Galaxy and also the regular magnetic field. These studies show that even at high latitudes, Galactic total and polarized emission is highly structured at arcsec resolution and must be separated from sensitive SKA maps of extended extragalactic objects in an appropriate way.

Studies of magneto-ionized turbulence through SFs will benefit greatly from a tight RM Grid as provided by SKA1. A grid with 1000 sources per square degree could unveil the second-order SF down to arcmin scales. The survey capability allows us to make the first detailed all-sky map of the energy injection scale, in order to reveal the origins of driving forces. Moreover, a large number of sources with small RM errors (e.g. $<1 \mathrm{rad} / \mathrm{m}^{2}$ ) allows us to derive higher-order SFs. Higher-order SFs provide further valuable information of turbulence, e.g. the trend of SF slopes to higher order depends on the characteristics of turbulence.

SKA1 pulsar studies will detect many more pulsars, including more DM measurements and reliable distances. The right hand plot in figure 1 shows the wavelet transform result of a test function representing electron density is obtained using a simulated pulsar data set of 2000 pulsars expected to be detected by SKA1 (R. Smits, priv. comm.). It is obvious from this plot that this method promises major progress in the unraveling of the large-scale structure of the Galactic magnetic field.

Supernova remnants With SKA1, the RM Grid will provide one source every few arcminutes. This means that we will be able to roughly probe the magnetic field of virtually every SNR in the Galaxy using the grid. Faraday tomography with SKA1-LOW/SKA1-SUR Band 1 will delineate magnetic fields in the SNR shell in 3D in pointed observations of individual target sources. Statistical samples of magnetic field structure in HII regions, PWNe, Planetary Nebulae can be built 
up with these data. Also, SKA1-LOW will enable a full magnetic field survey of nearby SNRs, but not until the enhanced sensitivity of SKA2 will we be able to probe magnetic fields in SNRs throughout the Milky Way in this way.

Star forming regions The high resolution of the SKA1 survey mentioned above can be used to unveil the non-thermal emission in star forming regions, reconciling the wider Galactic magnetic field with MHD models of star formation, protostellar accretion, disk coupling and jet launching. Emission is likely to be present at $\sim 50 \mu \mathrm{Jy}$ at $1000 \mathrm{MHz}$, with a spectral index of -1 (Ainsworth et al. 2014), i.e. a $10 \sigma$ detection. SKA1-MID can be used for follow-up to obtain the spectrum. Using the SKA1-MID sensitivity of $63 \mu \mathrm{Jy} \mathrm{hr}^{-1 / 2}$ over a $100 \mathrm{kHz}$ band, a spectrum with a $3 \sigma$ detection at $1 \mathrm{GHz}$ over $10 \mathrm{MHz}$ channels can be obtained in 30 mins per source. To date, two YSO jets have been detected in pointed observations (Ray et al. 1997; Carrasco-González et al. 2010), making it impossible to estimate reliable detection rates at the moment. We assume that we will be able to inspect the nearest star formation regions in total intensity with SKA1. Even a detection of a handful of YSO jets will make a large impact on the source sample.

Galactic Center The dense SKA1 RM Grid will allow unprecedented modeling of the structure and strength of the magnetic field in the GC, resolving the tension in the findings described in Section 2.3. In particular, detection of 15,000 new pulsars with measured RMs will allow us to understand the overall structure of the GC magnetic field (i.e., is it toroidal? Poloidal?) in a comprehensive manner, and how this connects to the overall Galactic magnetic field structure. Higher-order SFs will become possible due to the high source density in the SKA RM Grid, which will enable determination of hereto elusive properties of interstellar turbulence such as intermittency. The RM Grid will identify Faraday screens and clarify their role in the ISM and hopefully also their origin.

\subsection{Prospects for SKA2}

A tenfold increase in sensitivity of SKA2 at frequencies from $350 \mathrm{MHz}$ to $24 \mathrm{GHz}$ will allow extension of the all-sky broadband polarimetric survey at high sensitivity down to lower frequencies. This will make a Faraday tomography all-sky map of magnetized turbulence possible, allowing not only to fully characterize these Galactic magnetic fields, but also to describe in detail polarized foregrounds for various cosmological research areas such as the Epoch of Reionization. Also, this increase in sensitivity for SKA2 will enable larger samples of YSO's, making statistical studies possible, and detection of linear polarization, adding information on the orientation and ordering of the field. Finally, Faraday tomography for SNRs throughout the Milky Way can be done, giving a complete sample of 3D magnetic field structure in SNRs throughout the Milky Way.

For the RM Grid, SKA2 will provide us with accurate distances for just about any detectable pulsar in the Milky Way. The large increase of reliable pulsar RMs and distances will finally allow resolution of the issue of number and location of large-scale reversals in the Milky Way, thanks to the accurate parallax distances measured throughout a major part of the Galaxy. Wavelet analysis as in Stepanov et al. (2002) will be possible (a) for the entire Galaxy, and (b) down to scales of about $100 \mathrm{pc}$, which is comparable to some current estimates of the turbulence injection scale.

Additionally, the SKA2 phase will also allow a high angular and $\lambda^{2}$-space resolution observation of non-thermal radio filaments in the GC such as the Radio Arc, which possesses RMs of 
$\sim-3 \times 10^{3} \mathrm{rad} \mathrm{m}^{2}$ (Yusef-Zadeh et al. 1987) - some of the highest known in our Galaxy - which will allow a detailed modeling of their origin, as well as discovery of numerous others at lower surface brightness.

\section{References}

Ainsworth, R. E., Scaife, A. M. M., Ray, T. P., et al. 2014, ApJL, 792, 18

Akahori T., Ryu D., Kim J., \& Gaensler B. M. 2013, ApJ, 767, 150

Armstrong, J. W., Rickett, B. J., \& Spangler, S. R. 1995, ApJ, 443, 209

Arzoumanian, Z., Safi-Harb, S., Landecker, T. L., Kothes, R., \& Camilo, F. 2008, ApJ, 687, 505

Arzoumanian, Z., Gotthelf, E. V., Ransom, S. M., et al. 2011, ApJ, 739, 39

Beck, R., \& Gaensler, B. M. 2004, NAR, 48, 1289

Beck, R., Bomans, D., Colafrancesco, S., et al. 2015, "Structure, dynamical impact and origin of magnetic fields in nearby galaxies in the SKA era" in proc. Advancing Astrophysics with the Square Kilometre Array, PoS(AASKA15)094

Blandford \& Payne, 1982, MNRAS, 199, 883

Blasi, P. 2013, A\&AR, 21, 70

Braun, R. "SKA1 Imaging Science Performance", Document number SKA-TEL-SKO-DD-XXX Revision A Draft 2

Brentjens, M. A., \& de Bruyn, A. G. 2005, A\&A, 441, 1217

Brentjens, M. A. 2011, A\&A, 526, A9

Brown, J. C., Haverkorn, M., Gaensler, B. M., et al. 2007, ApJ, 663, 258

Burkhart, B., Lazarian, A., \& Gaensler, B. M. 2012, ApJ, 749, 145

Carrasco-González, C., Rodríguez, L. F., Anglada, G., et al. 2010, Science 330, 1209

Carretti, E., Crocker, R. M., Staveley-Smith, L., et al. 2013, Nat, 493, 66

Carretti, E. et al, in preparation

Chevalier, R. A. 2000, ApJL, 539, L45

Chuss, D. T., Davidson, J. A., Dotson, J. L., et al. 2003, ApJ, 599, 1116

Donnert, J., Dolag, K., Lesch, H., et al. 2009, MNRAS, 392, 1008

Crocker, R. M., 2013, ASSP, 34, 397

Crocker, R. M., \& Aharonian, F., 2011, Physical Review Letters, 106, 101102

Crocker, R. M., Jones, D. I., Aharonian, F., et al. 2011, MNRAS, 413, 763

Crocker, R. M., Jones, D. I., Melia, F., et al., 2010, Nature, 463, 65

Crutcher, R. M., Roberts, D. A., Mehringer, D. M., et al. 1996, ApJL, 462, L79

De Bruyn, A.G., Pizzo, R., 2014 A\&A, in press

de la Chevrotière, A., St-Louis, N., Moffat, A. F. J., \& the MiMeS Collaboration 2014, ApJ, 781, 73

Eatough, R. P., Falcke, H., Karuppusamy, R., et al. 2013, Nat, 501, 391

Elmegreen, B. G., \& Scalo, J. 2004, ARA\&A, 42, 211

Ferreira, J., Dougados, C., \& Cabrit, S. 2006, A\&A, 453, 785

Ferrière, K., \& Schmitt, D. 2000, A\&A, 358, 125

Ferrière, K. 2007, EAS Publications Series, 23, 3

Ferrière, K. 2009, A\&A, 505, 1183 
Ferrière, K., \& Terral, P. 2014, A\&A, 561, A100

Fish, V. L., Reid, M. J., Argon, A. L., et al. 2003, ApJ, 596, 328

Fletcher, A., Beck, R., Shukurov, A., et al. 2011, MNRAS, 412, 2396

Frick, P., Stepanov, R., Shukurov, A., et al. 2001, MNRAS, 325, 649

Gaensler, B. M. 1998, ApJ, 493, 781

Gaensler, B. M., Landecker, T. L., Taylor, A. R., \& POSSUM Collaboration 2010, Bulletin of the American Astronomical Society, 42, \#470.13

Gaensler, B. M., Haverkorn, M., Burkhart, B., et al. 2011, Nature, 478, 214

Gaensler. B., Agudo, I., Akahori, T. et al. 2015, "Broadband Polarimetry with the Square Kilometre Array: A Unique Astrophysical Probe" in proc. Advancing Astrophysics with the Square Kilometre Array, PoS(AASKA15)103

Gelfand, J., Breton, R., Ng, C. U. et al. 2015, "Pulsar Wind Nebulae in the SKA era" in proc. Advancing Astrophysics with the Square Kilometre Array, PoS(AASKA15)046

Gao, X. Y., Reich, W., Han, J. L., et al. 2010, A\&A, 515, A64

Govoni, F., Johnston-Hollitt, M., Agudo, I., et al., 2014, Cosmic Magnetism Science in the SKA1 Era, summary of the Cosmic Magnetism Assessment Workshop

Gray, A. D., Landecker, T. L., Dewdney, P. E., et al. 1998, Nat, 393, 660

Green, J. A., McClure-Griffiths, N. M., Caswell, J. L., et al. 2012, MNRAS, 425, 2530

Han, J.L., Van Straten, W., Lazio, T.J.W. et al., 2015, "Three-dimensional Tomography of the Galactic and Extragalactic Magnetoionic Medium with the SKA" in proc. Advancing Astrophysics with the Square Kilometre Array, PoS(AASKA15)041

Han, J. L., Manchester, R. N., Lyne, A. G., et al. 2006, ApJ, 642, 868

Han, J. L., \& Zhang, J. S. 2007, A\&A, 464, 609

Hanasz, M., Wóltański, D., \& Kowalik, K. 2009, ApJL, 706, L155

Hanayama, H., Takahashi, K., Kotake, K., et al. 2005, ApJ, 633, 941

Harvey-Smith, L., Gaensler, B. M., Kothes, R., et al. 2010, ApJ, 712, 1157

Harvey-Smith, L., Madsen, G. J., \& Gaensler, B. M. 2011, ApJ, 736, 83

Haverkorn, M., Katgert, P., \& de Bruyn, A. G. 2003, A\&A, 404, 233

Haverkorn, M., Gaensler, B. M., McClure-Griffiths, N. M., et al. 2006, ApJS, 167, 230

Haverkorn, M., Gaensler, B. M., Brown, J. C., et al. 2006, ApJL, 637, L33

Haverkorn, M., Brown, J. C., Gaensler, B. M., et al. 2008, ApJ, 680, 362

Haverkorn, M., 2014, in "Magnetic Fields in Diffuse Media", eds E. Gouveia dal Pino and A. Lazarian, Springer, arXiv:1406.0283

Heald, G., Beck, R., de Blok, E., et al., 2015, "Magnetic Field Tomography in Nearby Galaxies with the Square Kilometre Array" in proc. Advancing Astrophysics with the Square Kilometre Array, PoS(AASKA15) 106

Iacobelli, M., Haverkorn, M., \& Katgert, P. 2013, A\&A, 549, A56

Iacobelli, M., Burkhart, B., Haverkorn, M., et al. 2014, A\&A, 566, 5

Jansson, R., \& Farrar, G. R. 2012, ApJ, 757, 14

Jelić, V., de Bruyn, A. G., Mevius, M., et al. 2014 A\&A, 568, 101

Johnston-Hollitt, M., et al., 2015, "Using SKA Rotation Measures to Reveal the Mysteries of the Magnetised Universe" in proc. Advancing Astrophysics with the Square Kilometre Array, PoS(AASKA15)092 
Jones, D. I., Crocker, R., M., Reich, W., et al., 2012, ApJ, 747, 12.

Killeen, N. E. B., Lo, K. Y., et al. 1992, ApJ, 385, 585

Kothes, R., \& Brown, J.-A. 2009, IAU Symposium, 259, 75

Kothes, R., Fedotov, K., Foster, T. J., et al. 2006, A\&A, 457, 1081

Kothes, R., Landecker, T. L., Reich, W., et al. 2008, ApJ, 687, 516

Kramer, M. \& Stappers, B., 2015, "An overview of pulsar science with the SKA" in proc. Advancing Astrophysics with the Square Kilometre Array, PoS(AASKA15)036

Landecker, T. L., Reich, W., Reid, R. ., et al. 2010, A\&A, 520, A80

Law, C. J., Brentjens, M. A. \& Novak, G., 2011 ApJ, 731, 36

Lazar, M., Schlickeiser, R., Wielebinski, R., \& Poedts, S. 2009, ApJ, 693, 1133

Lazaryan, A. L., \& Shutenkov, V. P. 1990, Soviet Astronomy Letters, 16, 297

Livio, 2011, American Institute of Physics Conference Series, J. E. McEnery, J. L. Racusin, N.

Gehrels, eds. (2011), 1358, 329

Machida, M., Nakamura, K., Kudo, T., et al. 2013, ApJ, 764, 81

Malkov M. A., Diamond P. H., Drury L., et al. 2010, ApJ 721, 750

Mao, S. A., Gaensler, B. M., Haverkorn, M., et al. 2010, ApJ, 714, 1170

Mao, S. A., McClure-Griffiths, N. M., Gaensler, B. M., et al. 2012, ApJ, 755, 21

Men, H., Ferrière, K., \& Han, J. L. 2008, A\&A, 486, 819

Moss, D., \& Sokoloff, D. 2008, A\&A, 487, 197

Moss, D., Sokoloff, D., Beck, R., et al. 2010, A\&A, 512, A61

Moss, D., Stepanov, R., Arshakian, T. G., et al. 2012, A\&A, 537, A68

Nota, T., \& Katgert, P. 2010, A\&A, 513, A65

Novak, G., Chuss, D. T., Renbarger, T., et al. 2003, ApJL, 583, L83

Oppermann, N., Junklewitz, H., Robbers, G., et al. 2012, A\&A, 542, A93

Orlando \& Strong, 2013, Nuclear Physics B Proceedings Supplements 239, 64

O'Sullivan, S. P., Brown, S., Robishaw, T., et al. 2012, MNRAS, 421, 3300

Pacini, F., \& Salvati, M. 1973, ApJ, 186, 249

Ransom, R. R., Uyaniker, B., Kothes, R., et al. 2008, ApJ, 684, 1009

Ransom, R. R., Kothes, R., Wolleben, M., et al. 2010, ApJ, 724, 946

Ray, 2009, Revista Mexicana de Astronomia y Astrofisica Conference Series, 36, 179

Ray, T. P., Muxlow, T. W. B., Axon, D. J., et al. 1997, Nature 385, 415

Reich, W., Fürst, E., Reich, P., et al. 2004, The Magnetized Interstellar Medium, 45

Reynolds, S. P., Gaensler, B. M., \& Bocchino, F. 2012, SSR, 166, 231

Robishaw, T., Green, J., Surcis, G., et al. 2015, "Measuring Magnetic Fields Near and Far with the SKA via the Zeeman Effect" in proc. Advancing Astrophysics with the Square Kilometre Array, PoS(AASKA15) 110

Roy, S., Pramesh Rao, A., \& Subrahmanyan, R., 2005, MNRAS, 360, 1305.

Roy, S., Pramesh Rao, A., \& Subrahmanyan, R., 2008, A\&A, 478, 435.

Ruzmaikin, A., Sokolov, D., \& Shukurov, A. 1988, Nat, 336, 341

Schlickeiser, R., \& Felten, T. 2013, ApJ, 778, 39

Schnitzeler, D. H. F. M., Katgert, P., \& de Bruyn, A. G. 2007, A\&A, 471, L21

Schnitzeler, D. H. F. M. 2010, MNRAS, 409, L99

Simard-Normandin, M., \& Kronberg, P. P. 1980, ApJ, 242, 74 
Smits, R., Stappers, B., Kramer, M., Karastergiou, A. 2011a, "Pulsar Survey with SKA Phase I", Document number WP2-040.030.010-TD-003

Smits, R., Tingay, S. J., Wex, et al. 2011b, A\&A, 528, 108

Sofue, Y., Machida, M., \& Kudoh, T. 2010, PASJ, 62, 1191

Sokoloff, D.D., Shukurov, A. 1990, Nat, 347, 51

Stil, J., Wityk, N., Ouyed, R., et al. 2009, ApJ, 701, 330

Stil, J. M., Taylor, A. R., \& Sunstrum, C. 2011, ApJ, 726, 4

Stepanov, R., Frick, P., Shukurov, A., et al. 2002, A\&A, 391, 361

Su, M., Slatyer, T., \& Finkbeiner, D., 2010, ApJ, 724, 1044

Sun, X. H., Han, J. L., Reich, W., et al. 2007, A\&A, 463, 993

Sun, X. H., Reich, W., Waelkens, A., et al. 2008, A\&A, 477, 573

Sun, X. H., \& Reich, W. 2009, A\&A, 507, 1087

Sun, X.-H., \& Reich, W. 2010, Research in Astronomy and Astrophysics, 10, 1287

Taylor, A. R., Stil, J. M., \& Sunstrum, C. 2009, ApJ, 702, 1230

Taylor, A. R., \& Salter, C. J. 2010, Astronomical Society of the Pacific Conference Series, 438, 402

Uyaniker, B., Kothes, R., \& Brunt, C. M. 2002, ApJ, 565, 1022

Van Eck, C. L., Brown, J. C., Stil, J. M., et al. 2011, ApJ, 728, 97

Wang, L., XiaoHong, C., Hui, Z., et al., 2015, "Investigations of supernovae and supernova remnants in the era of SKA" in proc. Advancing Astrophysics with the Square Kilometre Array, PoS(AASKA15)064

Widrow, L. M. 2002, Reviews of Modern Physics, 74, 775

Wolleben, M., \& Reich, W. 2004, A\&A, 427, 537

Wolleben, M., Landecker, T. L., Carretti, E., et al. 2009, IAU Symposium, 259, 89

Wolleben, M., Fletcher, A., Landecker, T. L., et al. 2010, ApJL, 724, L48

Yusef-Zadeh, F., \& Morris, M., 1987, ApJ, 322, 721. 